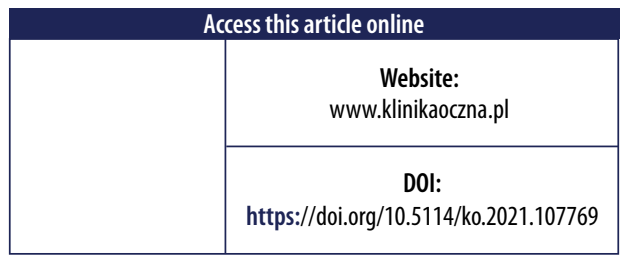

\title{
Adaptive optics imaging in the most common inherited retinal degenerations
}

\author{
Katarzyna Samelska, Magdalena Kupis, Anna Zaleska-Żmijewska, Jacek P. Szaflik \\ Department of Ophthalmology, Medical University of Warsaw, Poland \\ SPKSO Ophthalmic University Hospital, Warsaw, Poland
}

\begin{abstract}
Adaptive optics is the imaging technique of the retina. It finds use in visualizing microscopic changes in the retinal photoreceptors and the retinal blood vessels. Introducing adaptive optics as a diagnostic tool facilitated diagnosing inherited retinal diseases, such as Stargardt disease, cone-rod dystrophy, and retinitis pigmentosa These diseases are often inherited in a heterogenous pattern. Thus, genetic testing often fails to give a certain diagnosis. Optical coherence tomography, fundus autofluorescence, fluorescein angi-
\end{abstract}

ography, visual field examination and electrophysiological testing are vital for diagnosing patients with inherited retinal diseases. Adaptive optics imaging complements diagnosing and monitoring conditions such as Stargardt disease and cone-rod dystrophy. In this article we sum up the main characteristics of inherited retinal diseases in adaptive optics imaging.

KEY WORDS: adaptive optics, Stargardt disease, cone-rod dystrophy, retinitis pigmentosa.

\section{INTRODUCTION AND BASICS OF ADAPTIVE OPTICS}

Adaptive optics (AO) is a powerful tool in in vivo imaging of the microstructures of the retina. This imaging technique was primarily developed in astronomical telescopes in order to diminish atmospheric irregularities. The aberration-correcting system provided a high-quality image of distant objects [1].

The attempts to eliminate the optical aberrations of the eye were started by Dreher et al. [2]. The first use of AO in imaging the microstructures of the retina was reported in 1997 [3]. There are two main technologies of adaptive optics used in visualizing the retina's photoreceptors: split-detector (SD-AOSLO; split-detector adaptive optics scanning light ophthalmoscopy) and confocal (cSLO; confocal scanning laser ophthalmoscopy) - both confocal and spectral images may be taken simultaneously $[4,5]$

The classical retinal imaging adaptive optics camera consists of three main pieces: a wavefront sensor, a wavefront corrector and a control system. The aberrations are measured by a wavefront sensor and a corrector, whereas the controller interprets the sensor-collected data and controls the interaction between the sensor and corrector. There are two sources of light: one of them used to illuminate the retina and the second to measure and correct the wavefront aberrations $[1,4]$.
Lately, improved sensorless adaptive optics technology with enhanced quality parameters has been introduced [6]. The imaging is non-invasive and safe. It provides resolution of $2 \mu \mathrm{m}$. It may be performed with or without dilation of the patient's pupil; however, the pupil dilation is usually performed before the examination. The combination of $\mathrm{AO}$ and optical coherence tomography provides even greater resolution of the image and enables $3 \mathrm{D}$ visualization $[6,7]$.

\section{PRINCIPLES OF ADAPTIVE OPTICS IN OPHTHALMOLOGY}

One of the main goals of $\mathrm{AO}$ in ophthalmology is visualization of the photoreceptors: rods and cones. The photoreceptors' parameters examined in AOSLO are: cone density, cone spacing, Voronoi analysis, reflectivity, regularity, metrics for the preferred orientation of cones and local spatial anisotropy [5, 7-12]. The regions of photoreceptor loss allow visualization of the underlying retinal pigment epithelium (RPE) cells $[13,14]$. Photoreceptor pathologies are found in e.g. diabetic retinopathy $(\mathrm{DM})$ and age-related macular degeneration (AMD) $[15,16]$. Figure 1 shows an adaptive optics image of photoreceptors in a normal retina.

Adaptive optics provides, non-invasively, a precise image of the retinal microvasculature. Early changes in the microcirculation, e.g. in the course of DM and AMD, are detected 
in AO imaging $[4,15]$. The imaging of RNFL (retinal nerve fiber layer) and its glaucomatous changes is also a target of AOSLO - the image of hyperreflective bundles surrounded by dark lines depicts nerve fiber bundles and Muller cell septa [4].

Adaptive optics scanning laser ophthalmoscopy finds use in IRDs - inherited retinal degenerations. Photoreceptor visualization is a novel way to monitor the early and advanced stages of retinal diseases.

\section{CHARACTERISTICS OF INHERITED RETINAL DISEASES: STARGARDT DISEASE, CONE-ROD DYSTROPHY, RETINITIS PIGMENTOSA \\ Stargardt disease}

Stargardt disease is one of the most common inherited retinal diseases with a prevalence of around 1 in 10000 [5, 17]. It is a bilateral dystrophy which affects mainly the macu-

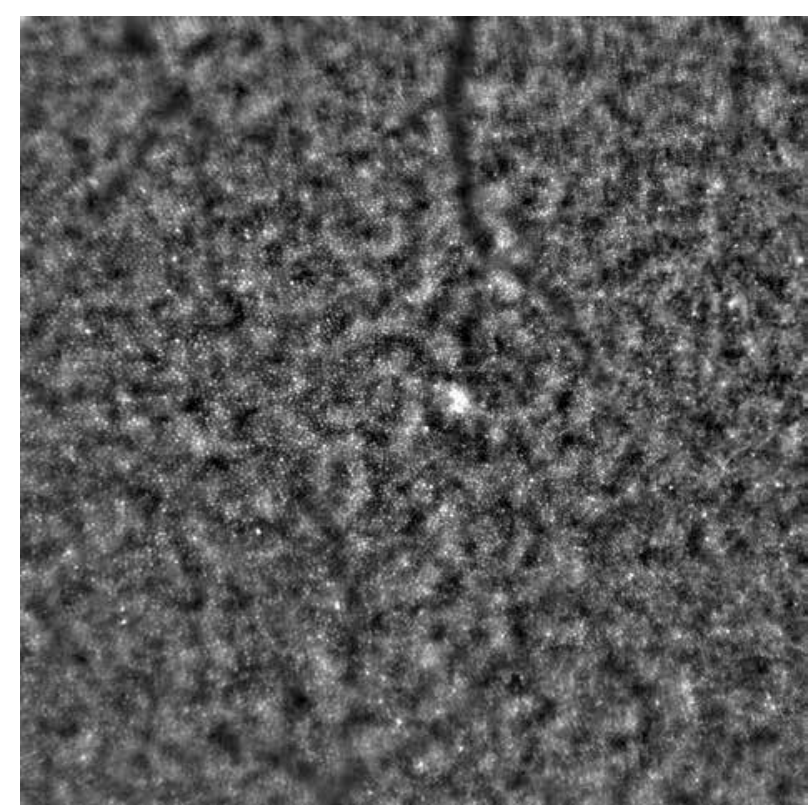

Figure 1. Photoreceptors in perifoveal area of a healthy eye imagined by adaptive optics

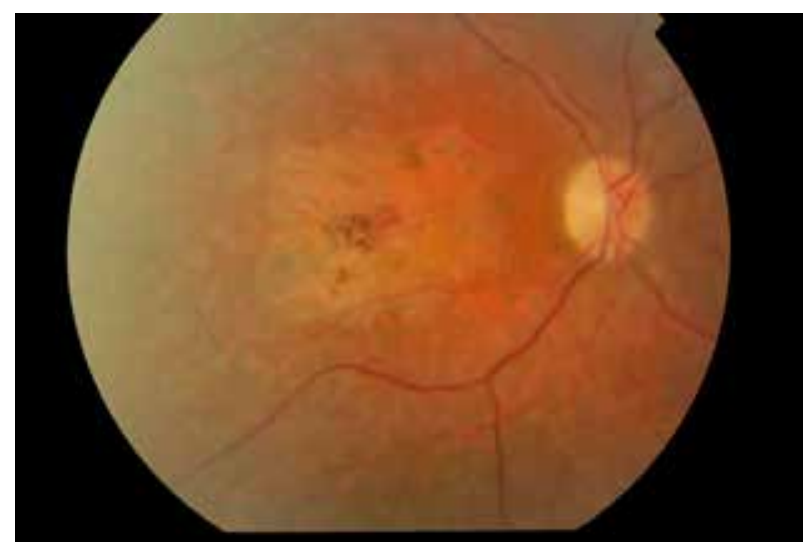

Figure 2. Stargardt disease: a photograph of the posterior pole la. It leads to loss of central vision and dyschromatopsia. The dynamics of vision deterioration differs individually depending on the location of the foveal lesion [18]. The presence of central scotoma is seen in visual field examination. Microperimetry be used for monitoring the progression of the disease [19]. Macular abnormalities in Stargardt disease are shown in Figure 2.

Stargardt maculopathy is a genetic condition in most cases associated with mutations in the ABCA4 gene (MIM601691) coding a protein located in outer segments of the photoreceptors. There are more than 200 identified pathogenic variants of ABCA4 mutations leading to Stargardt disease while the three most common mutations are responsible for only $27 \%$ of the cases [20]. SD-OCT (spectral-domain optical coherence tomography) shows changes in the central macula such as loss of the outer retina structure. The dynamics of Stargardt disease progression can be defined using SD-OCT through ellipsoid zone loss evaluation [21].

Fundus autofluorescence (FAF) shows loss of central autofluorescence in the central macula and an increased signal in surrounding regions resembling bulls-eye maculopathy. With the use of FAF testing we can monitor progression of the morphological changes of the macula. The mean progression of definitely decreased autofluorescence lesions is determined as 0.51-0.76 $\mathrm{mm}^{2} /$ year, and of total decreased FAF as $0.35 \mathrm{~mm}^{2} /$ year $[22,23]$. According to the FAF image characteristics of the fovea (including foveal sparing) and homogeneity of the background, Stargardt disease eyes may be divided into three subtypes. The RAE (rate of atrophy enlargement) differs between the three subtypes [24].

Fluorescein angiography (FFA), used more commonly in the past, showed a "dark choroid" sign in the central macula due to blocking of the choroid signal by lipofuscin deposits in the central macula. Electrophysiological tests, such as mfERG (multifocal electroretinography), show decreased macular function with a preserved peripheral signal $[5,17]$. An FFA image of Stargardt disease is shown in Figure 3.

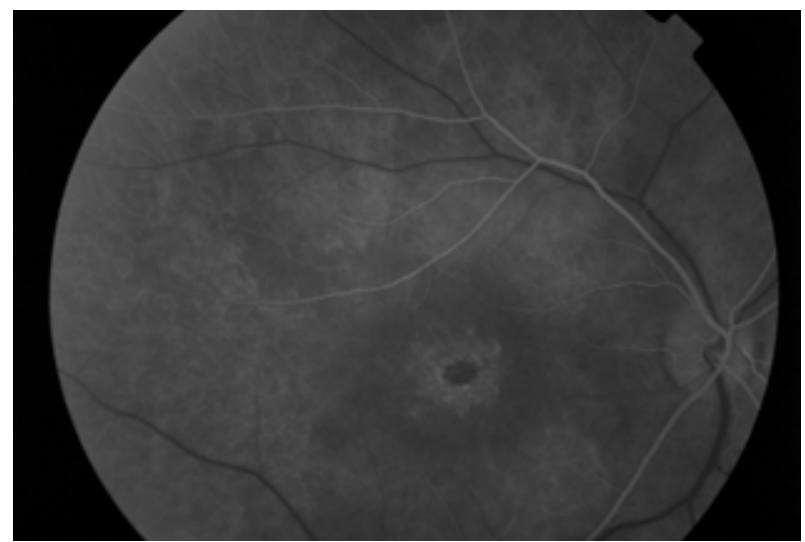

Figure 3. Stargardt disease: FFA image. Note the "dark choroid" sign in the central macula 


\section{Characteristics of adaptive optics imaging in Stargardt disease}

Spectral as well as confocal AO imaging is a potent tool in imaging pathologies in photoreceptors' morphology of eyes with Stargardt disease. The cone spacing is increased and cone density is decreased in Stargardt subjects' retina compared to normal retina $[5,17,25]$, as shown in Figure 4 . The confocal AO images show "dark spaces" within photoreceptors' structure, which represent non-wave guiding cones visualized in SD-AOSLO. The AOSLO changes may be found before the OCT and FAF detectable changes [25].

The development of the SD-AOSLO technique revealed that the cone density in Stargardt disease is in fact higher than was expected based on confocal AOSLO imaging and improves the quality of the imaging, making it more accurate in IRDs [5, 17].

There is a correlation between increased autofluorescence and loss of photoreceptor cells. It has been found however that AOSLO image reflectivity does not correlate with lipofuscin accumulation. AOSLO images show a hyperreflective perifoveal ring and its margin marks the margin of the scotoma [26].

Adaptive optics however meets some difficulties in Stargardt disease evaluation. The central scotoma and poor fixation make it unable to examine some eyes thoroughly. The accumulation of lipofuscin prevents AOSLO photoreceptor visualization in certain areas [27]. The cone spacing and density differ between the examined areas within the same eye and there has not been established a universal way to evaluate the degree of cone atrophy [26]. A possible solution may be creating cone density deviation (CDD) maps that present variation in foveal structure and may be compared with OCT and microperimetry results [28].

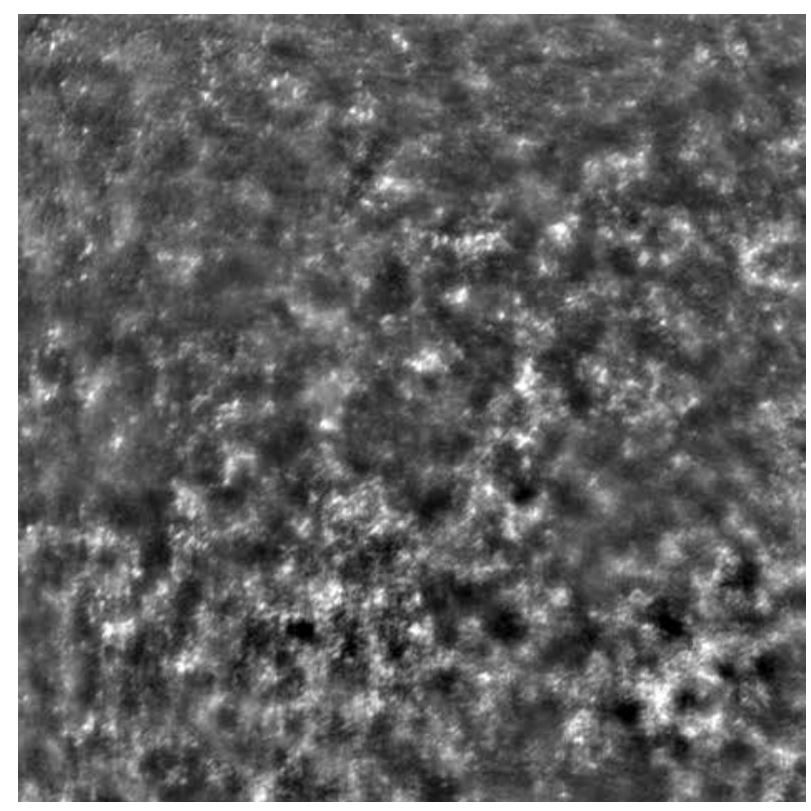

Figure 4. Stargardt disease: A0 image of the perifoveal photoreceptors. Note the dark spaces and decreased cone density
Adaptive optics enables visualization of the photoreceptors' morphology in Stargardt disease which complements FAF visualization of lipofuscin accumulation and OCT crosssection of the retinal layers [27].

\section{Cone-rod dystrophy}

Cone-rod dystrophy (CRD) is a retinal dystrophy where the dysfunction is higher in cones than in rods. The prevalence ranges from 1/30 000-1/40 000 [29]. It is genetically polymorphic with multiple variants of inheritance [30, 31]. The eye fundus examination shows perifoveal atrophy and "bull's eye" appearance (Figure 5). The changes include loss of RPE, photoreceptor loss and lipofuscin accumulation. The symptoms include photoaversion, progressive visual acuity deterioration and poor color vision due to cone dysfunction. In advanced stages, CRD leads to bilateral blindness [29, 31]. The diagnostic process of CRD includes genetic testing, OCT,

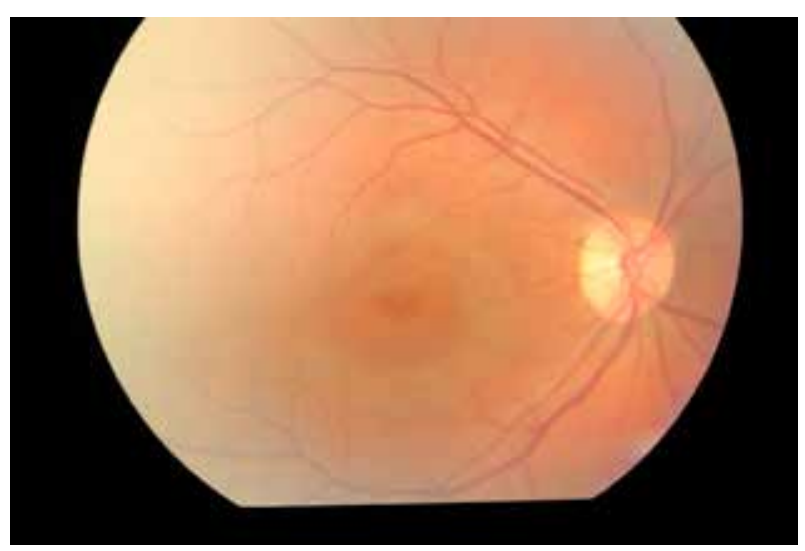

Figure 5. Cone-rod dystrophy: a photograph of the posterior pole

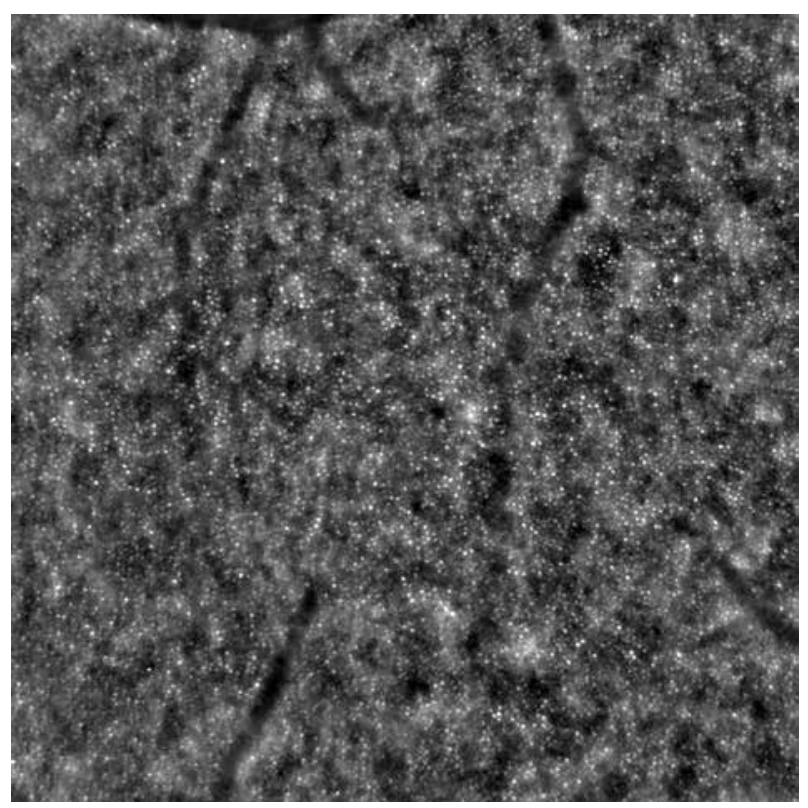

Figure 6. Cone-rod dystrophy: A0 image of the perifoveal photoreceptors. Note the decreased cone density 
ERG and FAF. The scotopic and photopic ERG cone and rod responses are extinguished in CRD [31].

\section{Characteristics of adaptive optics imaging in cone-rod dystrophy}

The photoreceptor AO imaging in CRD seen in AOSLO shows disruption of its mosaic and increased cone spacing and rod spacing, as seen in Figure 6. The level of the changes increases with the age of the subject. The photoreceptors in CRD show higher spacing, lower density, and lack of waveguiding cones within atrophic regions. The lowered-density regions' cone spacing measurements correlate with mfERG, microperimetry results and visual acuity changes [7, 13, 30, 32, 33].

The AOSLO imaging of the nine AD-CRD (autosomaldominant cone-rod dystrophy) confirmed patients from the same family showed that the pattern of photoreceptor damage varies between the subjects with the same type of mutation, regardless of age [34].

\section{Retinitis pigmentosa}

Retinitis pigmentosa (RP) is an inherited progressive bilateral degeneration of the photoreceptors. Like other IRDs mentioned above, the disease may be caused by mutations in multiple genes, with a heterogenous inheritance pattern [35]. The rod loss precedes the cones loss; the changes result in reduced visual acuity, a constricted visual field and, eventually, blindness [11, 32, 36, 37]. Retinitis pigmentosa may be isolated or a part of another syndrome, e.g. Usher syndrome, where sensorineural hearing loss is also present. The characteristics of RD in the ophthalmoscopic examination include presence of bone cells, which starts in the outer regions of the retina and proceeds to the macular region in more advanced stages, blood vessel narrowing, optic nerve atrophy and macular changes: signs of dystrophy or tapetal macular reflex. An eye fundus image of RP is shown in Figure 7 . The phenotype differs even within subjects with the same type of mutation [38].

Visual field examination shows progressive narrowing of the visual field. SD-OCT and time-domain OCT are used to

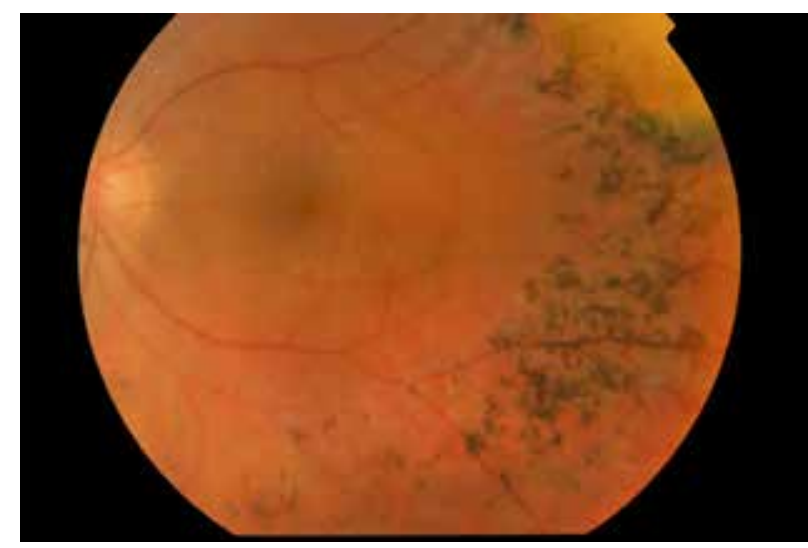

Figure 7. Retinitis pigmentosa: a photograph of the posterior pole visualize the photoreceptor layers. It shows thinning or loss of outer retinal layers throughout the macula and hyper-reflective profiles in the subretinal space at the fovea. However, it lacks resolution for single photoreceptor visualization and evaluation $[36,38]$. The ERG responses are distinguished, in more advanced stages, below the level of test reliability [38].

Changes in the macula in retinitis pigmentosa eyes are not uncommon. Diseases such as epiretinal membrane and macular hole may be found due to ischemia of macular RPE as well as changes in the vitreous body structure. Such conditions may be treated surgically [37, 39].

\section{Characteristics of adaptive optics imaging in retinitis} pigmentosa

Cone loss is observed in retinitis pigmentosa as well as Usher syndrome. The cone spacing in RP eyes is higher than in healthy eyes $[14,32,35,38]$. Regions of photoreceptor atrophy and hyper-reflective structures in outer retinal layers are visualized [38], as seen in Figure 8.

The interpretation of the images of the photoreceptors in retinitis pigmentosa shows a high level of variability between interpreters due to the cone loss areas in the retina. The image interpretation quality seems to be higher in SD-AOSLO compared to confocal AO $[5,40]$.

Foveal visual acuity loss and foveal sensitivity changes in retinitis pigmentosa are noted when morphological changes in the retina are already in an advanced stage [41].

The efforts to determine the rate of disease progression with AOSLO seem to be successful - it has been confirmed as consistent with the progression in SD-OCT and microperimetry findings [36] and as a successful tool in monitoring disease progression during experimental treatment [11].

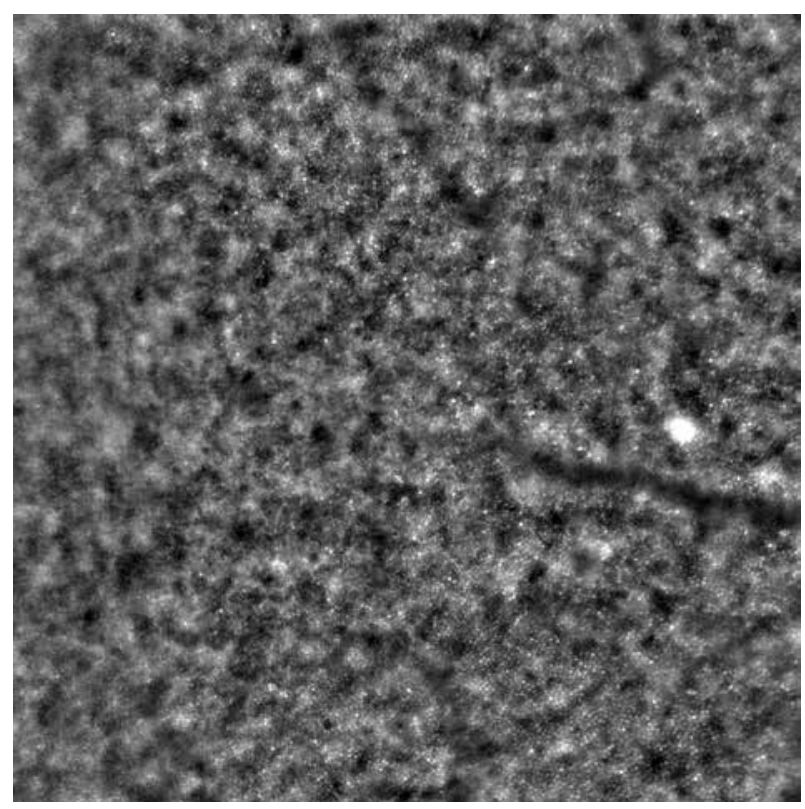

Figure 8. Retinitis pigmentosa: A0 image of the perifoveal photoreceptors. Note the dark spaces, decreased cone density and hyper-reflective structures 


\section{THE USE OF ADAPTIVE OPTICS IN OTHER INHERITED RETINAL DEGENERATIONS}

The AOSLO images of patients with BED (Bornholm eye disease), an X-linked cone dysfunction, prove the disruption of the cone mosaic with a high level of intersubject variability between patients with different disease-causing mutations [42].

Achromatopsia (ACHM) is also a subject of AO retinal studies. Split-detector images demonstrate a decreased number of photoreceptors as well as variations in cone density maps between the regions within the retina and between the eyes of ACHM subjects [9, 43].

The AO studies of patients with occult macular dystrophy (OMD) show regions of loss of photoreceptors, RPE visualization and lower cone density than in healthy eyes and a correlation of the morphological changes with the microperimetry [44-46].

\section{LIMITATIONS}

The challenges of adaptive optics include obtaining goodquality images in poor fixation, as well as in nystagmus - the eye tracking devices overcome those difficulties [47-49].

The interpretation of the images is also prone to mistakes made by non-experienced examiners. The machine learning of $\mathrm{AO}$ interpretation is vital to establish objective interpretation and forming right conclusions.

The need for larger study cohorts in IRDs is emphasized [50].

\section{SUMMARY}

Numerous ophthalmic researchers emphasize the role of longitudinal studies of patients with inherited retinal diseases. The research is limited by the low prevalence and difficult diagnostic process in some cases. The classical and well-known techniques, such as OCT, fundus autofluorescence, fluorescein angiography, and electroretinography used in diagnosing and monitoring IRD patients play a vital role. With the course of scientific development, the role of novel ophthalmic diagnostic solutions is becoming more significant. Adaptive optics imaging gives hopes for better understanding of the morphological basics and natural course of retinal diseases and with the introduction of experimental therapies also for monitoring the effectiveness of introduced treatment. Being able to monitor rare retinal conditions is crucial for the further development of medical solutions.

\section{DISCLOSURE}

The authors declare no conflict of interest.

\section{REFERENCES}

1. Gill JS, Moosajee M, Dubis AM. Cellular imaging of inherited retinal diseases using adaptive optics. Eye (Lond) 2019; 33: 1683-1698.

2. Dreher AW, Bille JF, Weinreb RN. Active optical depth resolution improvement of the laser tomographic scanner. Appl Opt 1989; 28: 804-808.

3. Liang J, Williams DR, Miller DT. Supernormal vision and high-resolution retinal imaging through adaptive optics. J Opt Soc Am A Opt Image Sci Vis 1997; 14: 2884-2892.

4. Lombardo M, Serrao S, Devaney N, et al. Adaptive Optics Technology for High-Resolution Retinal Imaging. Sensors (Basel) 2012; 13:334-366.

5. Tanna P, Kasilian M, Strauss R, et al. Reliability and repeatability of cone density measurements in patients with Stargardt disease and RPGR-associated retinopathy. Invest Ophthalmol Vis Sci 2017; 58: 3608-3615.

6. Polans J, Keller B, Carrasco-Zevallos OM, et al. Wide-field retinal optical coherence tomography with wavefront sensorless adaptive optics for enhanced imaging of targeted regions. Biomed Opt Express 2017; 8: 16-37.

7. Georgiou M, Kalitzeos A, Patterson EJ, et al. Adaptive optics imaging of inherited retinal diseases. Br J Ophthalmol 2018; 102: 1028-1035.

8. Zaleska-Żmijewska A, Wawrzyniak ZM, Ulińska M, et al. Human photoreceptor cone density measured with adaptive optics technology (rtx1 device) in healthy eyes: Standardization of measurements. Medicine (Baltimore) 2017; 96: e7300.

9. Dubis AM, Cooper RF, Aboshiha J, et al. Genotype-dependent variability in residual cone structure in achromatopsia: toward developing metrics for assessing cone health. Invest Ophthalmol Vis Sci 2014; 55: 7303-7311.

10. Godara P, Wagner-Schuman M, Rha J, et al. Imaging the photoreceptor mosaic with adaptive optics: beyond counting cones. Adv Exp Med Biol 2012; 723: 451-458.

11. Talcott KE, Ratnam K, Sundquist SM, et al. Longitudinal study of cone photoreceptors during retinal degeneration and in response to ciliary neurotrophic factor treatment. Invest Ophthalmol Vis Sci 2011; 52: 2219-2226.

12. Lombardo $M$, Serrao S, Lombardo $G$. Technical factors influencing cone packing density estimates in adaptive optics flood illuminated retinal images. PLoS One 2014; 9: e107402.

13. Roorda A, Zhang Y, Duncan JL. High-resolution in vivo imaging of the RPE mosaic in eyes with retinal disease. Invest Ophthalmol Vis Sci 2007; 48: 2297-2303.

14. Ratnam K, Västinsalo H, Roorda A, et al. Cone structure in patients with usher syndrome type III and mutations in the Clarin 1 gene. JAMA Ophthalmol 2013; 131: 67-74.

15. Zaleska-Żmijewska A, Wawrzyniak ZM, Dąbrowska A, et al. Adaptive Optics (rtx1) High-Resolution Imaging of Photoreceptors and Retinal Arteries in Patients with Diabetic Retinopathy. J Diabetes Res 2019; 2019: 9548324.

16. Zaleska-Żmijewska A, Piątkiewicz P, Śmigielska B, et al. Retinal Photoreceptors and Microvascular Changes in Prediabetes Measured with Adaptive Optics (rtx1 ${ }^{\left.{ }^{m}\right)}$ ): A Case-Control Study. J Diabetes Res 2017; 4174292.

17. Tanna P, Strauss RW, Fujinami K, et al. Stargardt disease: clinical features, molecular genetics, animal models and therapeutic options. Br J Ophthalmol 2017; 101: 25-30.

18. Kong X, Fujinami K, Strauss RW, et al. Visual Acuity Change Over 24 Months and Its Association with Foveal Phenotype and Genotype in Individuals With Stargardt Disease: ProgStar Study Report No. 10. JAMA Ophthalmol 2018; 136: 920-928. 
19. Schönbach EM, Strauss RW, Ibrahim MA, et al. Faster Sensitivity Loss around Dense Scotomas than for Overall Macular Sensitivity in Stargardt Disease: ProgStar Report No. 14. Am J Ophthalmol 2020; S0002-9394(20)30120-3.

20. Fujinami K, Strauss RW, Chiang JP, et al. Detailed genetic characteristics of an international large cohort of patients with Stargardt disease: ProgStar study report 8. Br J Ophthalmol 2019; 103:390-397.

21. Tanna P, Georgiu M, Strauss RW, et al. Cross-Sectional and Longitudinal Assessment of the Ellipsoid Zone in Childhood-Onset Stargardt Disease. TransI Vis Sci Technol 2019; 8: 1.

22. Strauss RW, Muñoz B, Ho A, et al. Progression of Stargardt Disease as Determined by Fundus Autofluorescence in the Retrospective Progression of Stargardt Disease Study (ProgStar Report No. 9). JAMA Ophthalmol 2017; 135: 1232-1241.

23. Strauss RW, Kong X, Ho A, et al. Progression of Stargardt Disease as Determined by Fundus Autofluorescence Over a 12-Month Period: ProgStar Report No. 11. JAMA Ophthalmol 2019; 137: 1134-1145.

24. Fujinami K, Lois N, Mukherjee R, et al. A longitudinal study of Stargardt disease: quantitative assessment of fundus autofluorescenCe, progression, and genotype correlations. Invest Ophthalmol Vis Sci 2013; 54: 8181-8190.

25. Song H, Rossi EA, Latchney L, et al. Cone and rod loss in Stargardt disease revealed by adaptive optics scanning light ophthalmoscopy. JAMA Ophthalmol 2015; 133: 1198-1203.

26. Chen Y, Ratnam K, Sundquist SM, et al. Cone photoreceptor abnormalities correlate with vision loss in patients with Stargardt disease. Invest Ophthalmol Vis Sci 2011; 52: 3281-3292.

27. Chen Y, Roorda A, Duncan JL. Advances in imaging of Stargardt disease. Adv Exp Med Biol 2010; 664: 333-340.

28. Razeen MM, Cooper RF, Langlo CS, et al. Correlating photoreceptor mosaic structure to clinical findings in Stargardt disease. Transl Vis Sci Technol 2016; 5: 6.

29. Hamel CP, Griffoin JM, Bazalgette C, et al. Molecular genetics of pigmentary retinopathies: identification of mutations in CHM, RDS, RHO, RPE65, USH2A and XLRS1 genes. J Fr Ophtalmol 2000; 23: 985-995.

30. Wolfing Jl, Chung M, Carroll J, et al. High-resolution retinal imaging of cone-rod dystrophy. Ophthalmology 2006; 113: 1014-1019.

31. Wawrocka A, Skorczyk-Werner A, Wicher K, et al. Novel Variants Identified with Next-Generation Sequencing in Polish Patients With Cone-Rod Dystrophy. Mol Vis 2018; 24: 326-339.

32. Duncan JL, Zhang Y, Gandhi J, et al. A. High-Resolution imaging with adaptive optics in patients with inherited retinal degeneration. Invest Ophthalmol Vis Sci 2007; 48: 3283-3291.

33. Choi SS, Doble N, Hardy JL, et al. In vivo imaging of the photoreceptor mosaic in retinal dystrophies and correlations with visual function. Invest Ophthalmol Vis Sci 2006; 47: 2080-2092.

34. Song H, Rossi EA, Stone E, et al. Phenotypic diversity in autosomal-dominant cone-rod dystrophy elucidated by adaptive optics retinal imaging. Br J Ophthalmol 2018; 102: 136-141.

35. Park SP, LeeW, Bae EJ, et al. Early structural anomalies observed by high-resolution imaging in two related cases of autosomal-dominant retinitis pigmentosa. Ophthalmic Surg Lasers Imaging Retin 2014; 45: 469-473.

36. Foote KG, De la Huerta I, Gustafson K, et al. Cone Spacing Correlates With Retinal Thickness and Microperimetry in Patients With Inherited Retinal Degenerations. Invest Ophthalmol Vis Sci 2019; 60: 1234-1243.

37. Nowosielska A. Vitrectomy for macular lesions in eyes with retinitis pigmentosa. Klin Oczna 2018; 3: 155-158.

38. Duncan JL, Biswas P, Kozak I, et al. Ocular phenotype of a family with FAM161A-associated retinal degeneration. Ophthalmic Genet 2016; 37: 44-52.

39. Jin ZB, Gan DK, Xu GZ, et al. Macular hole formation in patients with retinitis pigmentosa and prognosis of pars plana vitrectomy. Retina 2008; 28: 610-614.

40. Giannini D, Lombardo G, Mariotti L, et al. Reliability and agreement between metrics of cone spacing in adaptive optics images of the human retinal photoreceptor mosaic. Invest Ophthalmol Vis Sci 2017; 58: 3127-3137.

41. Ratnam K, Carroll J, Porco TC, et al. Relationship between foveal cone structure and clinical measures of visual function in patients with inherited retinal degenerations. Invest Ophthalmol Vis Sci 2013; 54: 5836-5847.

42. Patterson EJ, Tee J, Neitz J, et al. Assessing cone mosaic disruption in patients with X-linked cone dysfunction. Invest Ophthalmol Vis Sci 2015; 56: 88-88.

43. Litts KM, Georgiou M, Langlo CS, et al. Interocular Symmetry of Foveal Cone Topography in Congenital Achromatopsia. Curr Eye Res 2020;13:1-8.

44. Nakanishi A, Ueno $S$, Kawano $K$, et al. Pathologic changes of cone photoreceptors in eyes with occult macular dystrophy. Invest Ophthalmol Vis Sci 2015; 56: 7243-7249.

45. Tojo N, Nakamura T, Ozaki H, et al. Analysis of macular cone photoreceptors in a case of occult macular dystrophy. Clin Ophthalmol 2013; 7:859-864.

46. Viana KI, Messias A, Siqueira RC, et al. Structure-functional correlation using adaptive optics, OCT, and microperimetry in a case of occult macular dystrophy. Arq Bras Oftalmol 2017; 80: 118-121.

47. Sheehy CK, Tiruveedhula P, Sabesan R, et al. Active eye-tracking for an adaptive optics scanning laser ophthalmoscope. Biomed Opt Express 2015; 7: 2412-2423.

48. Privitera $\mathrm{CM}$, Sabesan R, Winter S, et al. Eye-tracking technology for real-time monitoring of transverse chromatic aberration. Opt Lett 2016; 41: 1728-1731.

49. Tam J, Roorda A. Speed quantification and tracking of moving objects in adaptive optics scanning laser ophthalmoscopy. J Biomed Opt 2011; 16:036002.

50. Strauss RW, Ho A, Munoz B, et al. The Natural History of the Progression of Atrophy Secondary to Stargardt Disease (ProgStar) Studies: Design and Baseline Characteristics: ProgStar Report No. 1. Ophthalmology 2016; 123: 817-828. 